\title{
Überwässerung und Dialyse beim akuten Nierenversagen
}

Fuat H. Saner, Anja Bienholz, Bartosz Tyczynski, Andreas Kribben, Thorsten Feldkamp

Bis zu $80 \%$ der Patienten mit einem dialysepflichtigen akuten Nierenversagen sterben noch auf der Intensivstation. Ein wichtiger Prädiktor für die Sterblichkeit ist Überwässerung: Ist der Patient überwässert, führt die Dilution zu einem erniedrigten Kreatininwert, der die Diagnose verschleiern kann. Bei jedem dieser kritisch Kranken sollte ein interdisziplinäres Team alle Umstände abwägen und entscheiden, ob und zu welchem Zeitpunkt der Patient eine Dialyse erhalten wird.

\begin{abstract}
Akutes Nierenversagen I Das akute Nierenversagen (ANV) ist ein klinisches Syndrom, dessen Genese ein äußerst weites und heterogenes Spektrum umfasst. Definiert ist das ANV als rascher Abfall der glomerulären Filtrationsrate (GFR), signalisiert durch einen Anstieg des Serumkreatinins und/oder eine Einschränkung der Diurese. Die häufigste Ursache des ANV ist eine direkte ischämisch-toxische Nierenschädigung oder eine zur Aufrechterhaltung der GFR unzureichende renale Perfusion. Jede Form des Schocks kann letzteres auslösen, wobei auf der Intensivstationen die Sepsis eine besondere Rolle spielt. Ein postrenales Nierenversagen muss man zwingend ausschließen [19]. So kann ein obstruierter Blasenkatheter auch eine Ursache für ein ANV sein.
\end{abstract}

Ein ANV liegt vor, wenn mindestens eines der folgenden Kriterien erfüllt wird [6]:

- Anstieg des Serumkreatinins um mindestens $0,3 \mathrm{mg} / \mathrm{dl}$ innerhalb von $48 \mathrm{~h}$

- Anstieg des Serumkreatinins auf mindestens das 1,5-Fache eines bekannten oder angenommenen Ausgangswerts innerhalb von 7 Tagen

- Abfall der Urinausscheidung auf weniger als $0,5 \mathrm{ml} / \mathrm{kg}$ Körpergewicht / h für mindestens $6 \mathrm{~h}$

- Basierend auf diesen Veränderungen kann eine Schweregradeinteilung des ANV erfolgen $(\triangleright$ Tab. 1).

Flüssigkeitstherapie | Bis vor wenigen Jahren war beim oligurischen ANV die Zufuhr kristalliner Flüssigkeiten das Mittel der Wahl. Dies beruhte auf der Vorstellung, der Niere ausreichend Flüssigkeit anzubieten, um so die Perfusion wieder zu verbessern. Bei Patienten im frühen Stadium eines ANV aufgrund einer Volumendepletion funktioniert dies gut. Sollte die Niere aber bereits strukturell geschädigt und nicht in der Lage sein, die angebotene Flüssigkeit auszuscheiden, droht eine Überwässerung [18]. Es konnte gezeigt werden, dass solch eine Überwässerung im Rahmen des ANV zu generalisierten Ödemen mit Gewebshypoxie führt - auch in der Niere selbst [39]. Dies führt zu einer weiteren Nierenschädigung und Funktionsverlust.
Der Funktionsverlust eines Organs aufgrund eines Ödems kann man am eindrucksvollsten an der Lunge verfolgen. Hier konnte man mit einer restriktiven Flüssigkeitstherapie die Beatmungszeit deutlich verkürzen, ohne die Rate der Nierenschädigung zu steigern $[20,47]$.

Bei einem oligurischen ANV ist es daher sinnvoll, eine Dialysetherapie zu einem Zeitpunkt zu beginnen, an dem sich noch kein sekundäres Gewebeödem entwickelt hat.

\section{Überwässerung - verzögerte Diagnose des ANV}

Verdünntes Serumkreatinin I Die Diagnose des ANV stützt sich neben der Urinproduktion auf den Anstieg des Serumkreatinins [26]. Die aktuellen KDIGO-Leitlinien (Kidney Disease - Improving Global Outcomes) für die Diagnostik des ANV unterscheiden drei verschiedene Schweregrade, basierend auf Urinausscheidung und Serumkreatinin (SCr) ( $\triangleright$ Tab. 1) [6]. In verschiedenen Untersuchungen konnte gezeigt werden, dass selbst ein ANV Grad I - d. h. ein Anstieg des Serumkreatinins von mindestens $0,3 \mathrm{mg} / \mathrm{dl}$ - mit einem erheblich erhöhten Mortalitätssrisiko einhergeht [12, 28, 36]. Die Diagnose des ANV wird häufig verschleiert, weil das Serumkreatinin im Rahmen der Überwässerung verdünnt ist. Folglich können die behandelnden Ärzte nephroprotektive Maßnahmen nicht einleiten oder renal eliminierte Medikamente nicht in ihrer Dosis an die verringerte Nierenfunktion anpassen.

Adjustierter Serumkreatininwert | Macedo et al. führte eine retrospektive Datenanalyse an dem Patientenkollektiv, das für die PICARD Studie rekrutiert worden war [30, 32]. Auf der Grundlage eines Algorithmus konnte aus den

- Rohdaten,

- dem Serumkreatinin und

- dem Grad der Überwässerung 
der um die Dilution adjustierte Serumkreatininwert erfasst werden. Es zeigte sich, dass in $25 \%$ der Fälle die Diagnose eines ANV nicht gestellt wurde, weil das Serumkreatinin durch Dilution verringert war [40]. Weiterhin untersuchten Liu et al. in einer Studie mit 1000 ARDS-Patienten die Inzidenz des ANV in den ersten 8 Tagen nach Aufnahme auf die Intensivstation anhand des Serumkreatinins. Dabei verwendete sie zur Diagnose einmal

- den reinen Serumkreatininwert und im Vergleich dazu

- den um die Dilution adjustierten Serumkreatininwert aufgrund des vergrößerten Extrazellulärraums [29].

Die Letalität der Patienten, die nach der Adjustierung ein ANV aufwiesen, war deutlich höher als die Letalität der Patienten ohne ANV vor und nach Adjustierung des Serumkreatinins (31\% vs. $12 \%$, $\mathrm{p}<0,001$ ). Die Letalität der Patienten mit einem ANV vor und nach Adjustierung war vergleichbar mit den Patienten, die nur nach der Adjustierung ein ANV hatten (38\% vs. $31 \%, p=0,18$ ).

Aus diesen Ergebnissen kann geschlossen werden, dass die Überwässerung der Patienten die Diagnose des ANV verschleiert, und dass auch dieses verschleierte ANV die Mortalität erhöht und somit große klinische Relevanz hat.

\section{Auswirkungen der Überwässerung}

Auswirkung auf den Gesamtorganismus | Die Arbeitsgruppe um Payen et al. hat sich mit dem Zusammenhang von Überwässerung und Überleben auseinandergesetzt [38]. Sie kamen zu dem Schluss:

Die Überwässerung ist ein unabhängiger Prädiktor hinsichtlich der Letalität bei septischen Patienten.

Eine andere Arbeitsgruppe kam zu einem ähnlichen Ergebnis: Die Erhebung von Bouchard et al. belegt, dass eine Flüssigkeitszunahme um 10\% gegenüber dem Ausgangsgewicht mit einer deutlich höheren 30- und 60-Tage- Krankenhausletalität assoziiert ist [7]. Die Odds Ratio (OR) hinsichtlich der Letalität beträgt bei den Dialysepatienten 2,07 (95\% KI 1,27-3,37), wenn die Dialysebehandlung erst bei Überwässerung begonnen wurde. Bei nicht-dialysierten, überwässerten Patienten betrug die OR sogar 3,14 (95\% KI 1,18$8,33)$. Aber auch andere Endpunkte wie

- Beatmungszeit und

- Inzidenz für Sepsis

waren bei überwässerten Patienten statistisch signifikant höher. In einer weiteren Untersuchung von Bellomo et al. an 1453 Patienten mit dialysepflichtigem ANV war eine durchschnittliche negative Flüssigkeitsbilanz von $-234 \mathrm{ml} /$ Tag gegenüber einer Bilanz von $+560 \mathrm{ml} /$ Tag mit einem

\section{Grad}

1

Anstieg um $\geq 0,3 \mathrm{mg} / \mathrm{dl}$ oder 1,5-1,9-Fache des Ausgangswertes

2 Anstieg auf das 2,0-2,9-Fache des Ausgangswerts

3 Anstieg auf das $\geq 3,0$-Fache des Ausgangswerts oder Anstieg auf $\geq 4,0 \mathrm{mg} / \mathrm{dl}$ oder Beginn einer Nierenersatztherapie

\section{Urinproduktion}

$<0,5 \mathrm{ml} / \mathrm{kg} \mathrm{KG} / \mathrm{h}$ für $6-12 \mathrm{~h}$

$<0,5 \mathrm{ml} / \mathrm{kg}$ KG $/ \mathrm{h}$ für $\geq 12 \mathrm{~h}$

$<0,3 \mathrm{ml} / \mathrm{kg} \mathrm{KG} / \mathrm{h}$ für $\geq 24 \mathrm{~h}$ oder Anurie für $\geq 12 \mathrm{~h}$ besseren Überleben assoziiert [4]. Die Autoren Tab. 1 Gradeinteilung des akuten Nierenversagens (ANV), modifiziert nach [6]. konnten zeigen, dass eine negative Flüssigkeitsbilanz ein unabhängiger Prädiktor für eine verringerte 90-Tage-Letalität mit einer OR von 0,318 (95\% KI 0,23-0,43) war. Diese Daten belegen eindeutig, dass eine Überwässerung zu einer Erhöhung der Mortalität führt.

Die andauernde Evaluation des Flüssigkeitsstatus ist auf der Intensivstation von entscheidender Bedeutung für die Prognose.

Auswirkungen auf die Niere | Die Überwässerung führt neben einer verzögerten Diagnose des ANV auch selbst zur Nierenschädigung. Auch wenn bislang kein direkter Nachweis gelang, werden zwei Ursachen diskutiert, die die Niere potenziell direkt schädigen können [22]:

1. erhöhter intraabdomineller Druck (IAP)

2. erhöhtes Risiko einer Sepsis bei ANV

Eine Überwässerung kann zu einem erhöhten IAP bis hin zum abdominellen Kompartment führen. Beides erhöht Morbidität und Letalität bei Patienten auf der Intensivstation [34]. Der erhöhte IAP führt zur

- Kompression der intraabdominellen Gefäße,

- Mikrozirkulationsstörungen sowie

- venösen Druckerhöhung im Bereich der V. renalis $[11,16]$.

Folglich fällt die glomeruläre Filtrationsrate(GFR), eine Oligurie entsteht [9]. Beim abdominellen Kompartment kommt es dann zu

- einem Abfall des Herzzeitvolumens (HZV),

- einer Aktivierung des Renin-Angiotensin-Aldosteron-Systems (RAAS) sowie

- des sympathischen Nervensystems.

Dies bewirkt eine renale Vasokonstriktion und verstärkt damit die renale Dysfunktion weiter [33]. Mehta et al konnte erstmals zeigen, dass ein ANV mit einer erhöhten Sepsisrate assoziiert ist [31]. Als Ursache führen die Autoren ein Darmödem aufgrund der Überwässerung an. Hierbei soll es zu einem Verlust der Barrierefunktion des Darms mit sekundärer Einschwemmung von Bakterien kommen, die dann über eine Bakteriämie wieder die Niere schädigen.

Chronifizierung | Patienten mit einem dialysepflichtigem ANV können je nach renaler Vorschä- 
digung in bis zu 15-20\% der Fälle eine terminale Nierenkrankheit erleiden $[21,46]$.

Ein akutes Nierenversagen erhöht das Risiko für eine chronische Nierenkrankheit.

Hier konnten weitere patienten-assoziierte Risikofaktoren wie z.B. Alter und verschiedene Komorbiditäten als Risikofaktoren identifiziert werden. Daten hinsichtlich Überwässerung und Nierenfunktion wurden in dieser Arbeit jedoch nicht erhoben [21]. Zu einer der ersten klinischen Arbeiten, die dies untersucht hat, zählt die Erhebung aus der Arbeitsgruppe Heung et al. [23]. Diese untersuchte retrospektiv 170 Patienten mit einem dialysepflichtigen ANV. Primärer Endpunkt war eine Erholung der Nierenfunktion mit dauerhafter Dialysefreiheit. Diesen primären Endpunkt erreichten innerhalb des ersten Jahres 35,9\% (61 / 170) der Patienten [26]. Die mediane Dialysedauer betrug 11 Tage. 83,6\% der 61 Patienten erreichten den primären Endpunkt noch vor der Entlassung aus dem Krankenhaus. Patienten, deren Nierenfunktion sich erholte, waren zum Zeitpunkt des Dialysebeginns deutlich weniger überwässert als Patienten, die dialysepflichtig blieben oder gar starben [23]. Dies untermauert die Hypothese, dass eine Überwässerung für die Erholung der Nierenfunktion ungünstig ist.

\section{Nierenersatztherapie: der richtige Zeitpunkt}

Dialyse - ja oder nein? | Als Kliniker ist man immer wieder mit der Frage konfrontiert, ob ein Patient mit ANV eine Dialysebehandlung erhalten soll. Die Acute Dialysis Quality Initiative (ADQI) hat vor mehr als einem Jahrzehnt absolute Indikationen für eine Dialyse erarbeitet. Diese beruhten jedoch nicht auf evidenzbasierten Daten, sondern waren eine Expertenmeinung [44]. Die KDIGOGruppe hat diese Indikationen 2012 erneut evaluiert und bewertet. Demnach sind folgende Bedingungen eine absolute Indikation zur Einleitung einer Dialysetherapie [1]:

1. urämische Komplikationen (Enzephalopathie, Perikarditis, Blutung)

2. Hyperkaliämie

3. schwere Azidose

4. Diuretika-resistentes Lungenödem

RIFLE-Kriterien I Die Forschungsaktivitäten etablierten eine abgestufte Klassifizierung des ANV, die die ADQI-Gruppe entwickelte und die weitgehend auch bei der neueren KDIGO-Klassifikation Verwendung findet. Dies sind die sogenannten RIFLE-Kriterien:

- Risk

- Injury

- Failure

- Loss of Function

- End-Stage-Renal Disease
Sie erlauben eine Graduierung des ANV und somit eine bessere Einschätzung hinsichtlich verschiedenen Stadien [5, 14]. Die RIFLE-Kriterien wurden zwar nicht entwickelt, um das Überleben vorherzusagen oder um eine bestimmte Therapie ab einem bestimmten Stadium einzuleiten. Dennoch berichten zwei Studien, dass sie Risikopatienten identifizieren und eine Voraussage hinsichtlich des Langzeitüberlebens bis zu 10 Jahren sowohl bei Patienten mit einer ambulant erworbenen Pneumonie als auch bei kardiochirurgischen Patienten liefern können [24, 35].

Abwägen aller Umstände | In der Literatur gibt es Hinweise, dass der Beginn einer Nierenersatztherapie im RIFLE-Stadium R oder I für den Patienten von Vorteil sein kann - insbesondere dann, wenn sich das ANV trotz adäquater Therapie rasch progredient vom Stadium I nach F bewegt [3]. Trotzdem kann man im Stadium I keine generelle Therapieempfehlung ableiten.

Bei jedem Patienten muss man die Dialyseindikation sehr genau abwägen und die gesamten Umstände in Betracht ziehen.

Wichtige Faktoren für die Indikation | Laut den KDIGO-Kriterien sollten bei der Indikationsstellung klinische Eckpunkte eine größere Rolle spielen als nur die Laborwerte von Serumkreatinin und Harnstoff-N [1]. So kann ein gewisser Vorschaden der Niere den rechtzeitigen Beginn einer Dialysebehandlung beeinflussen. Aber auch Begleitumstände wie

- Sepsis,

- septischer Schock mit erhöhter Kapillardurchlässigkeit (capillary leak),

- Polytrauma,

- obere GI-Blutung mit hohen Harnstoffwerten und

- Rhabdomyolyse

sind entscheidende Faktoren bei der Entscheidung, ob eine Dialyse indiziert ist. Nach den RIFLEKriterien könnte man einen Dialysebeginn im Stadium R als früh und Stadium F als spät werten.

Harnstoffkonzentration I Wie sind jedoch die Begleitumstände zu werten? Ist bei einem polytraumatisierten Patienten mit Massentransfusion das Stadium I das gleiche wie bei einem Patienten mit hydropisch dekompensierter Zirrhose oder einem Patienten mit Sepsis? Würde man hier eher den polytraumatisierten Patienten dialysieren oder eher den Patienten mit der dekompensierten Zirrhose? Diese Fragen sind mit den jetzigen Methoden nicht zu beantworten. Nichtsdestotrotz existieren Studien, die einen frühen mit einem späten Dialysebeginn verglichen haben - z. B. gemessen an der Harnstoffkonzentration. In einer retrospektiven Erhebung konnte man bei einem septischen Patientenkollektiv zeigen: 
Eine Dialyse war bei Patienten mit niedriger Harnstoffkonzentration nach septisch bedingtem ANV mit einem besseren Überleben assoziiert als bei Patienten mit einer hohen Harnstoffkonzentration.

Dieser Effekt konnte sowohl bei der 28-Tage-Mortalität als auch nach 365 Tagen gezeigt werden [10]. In einer anderen Erhebung wurde die gleiche Fragestellung bei operativen Patienten evaluiert. Dort untersuchte man das Überleben von Patienten mit postoperativer Leber- und Nierenschädigung bei hoher und niedriger Harnstoffkonzentration zum Dialysebeginn [48]. Hier zeigte sich, dass die hohe Harnstoffkonzentration unabhängig von anderen Faktoren mit einer erhöhten Mortalität assoziiert war (OR 4,01; KI 1,05-15,27). Diese Befunde konnte eine prospektiv randomisierte Studie nicht bestätigen - hier war ein Dialysebeginn bei niedriger Harnstoffkonzentration nicht mit besserem Überleben assoziiert [8].

Studien zum Dialysebeginn I Es wurden auch Studien durchgeführt, in denen der Dialysezeitpunkt durch die RIFLE-Kriterien definiert wurde. Hier zeigte sich in einem allgemeinchirurgischen Patientenkollektiv, dass das Risiko nach ANV zu versterben, bei Dialysebeginn in höheren RIFLEStadien 1,846-fach $(\mathrm{HR}=1,846)$ erhöht ist [42]. Diese Ergebnisse konnte jedoch eine andere retrospektive Studie mit Patienten nach septischem ANV nicht bestätigen [13]. In einer weiteren Studie aus China untersuchten Shum et al. an 120 Patienten mit schwerer Sepsis und ANV den Zeitpunkt der Dialyse hinsichtlich des Überlebens [43]. Das Nierenversagen beurteilten sie hinsichtlich der RIFLE-Kriterien. Der Zeitpunkt der Dialyse wurde wie folgt eingestuft:

- frühe Dialyse bei Patienten, die bereits im Stadium R eine Dialyse erhielten

- späte Dialyse bei Patienten, die erst im Stadium I oder F dialysiert wurden
In dieser Erhebung konnte kein Vorteil der frühen Dialyse gezeigt werden. Erwähnenswert ist, dass die Patienten in der späten Phase nach Aufnahme auf die Intensivstation deutlich früher dialysiert wurden als Patienten in der früheren Phase. Zusätzlich hatten die Patienten eine signifikant schlechtere Nierenfunktion, die spät dialysiert wurden. Aus diesen Gründen sind die Ergebnisse der Studie sicherlich verfälscht. Eine Metanalyse von Karvellas et al. konnte einen Überlebensvorteil der frühen Dialyse hinsichtlich der 28-TageLetalität herausarbeiten [25]. Karvellas et al. kombinierten Studien, in denen

- Harnstoff,

- Serumkreatinin,

- RIFLE-Kriterien und

- Urin-Ausscheidung

als Kriterien für den Dialysebeginn benutzt wurden - dies schränkt die Vergleichbarkeit der Studien ein. Zusätzlich wiesen die Autoren darauf hin, dass die ausgewählten 15 Studien zum Teil erhebliche methodische Mängel aufwiesen. Aus dieser Metaanalyse kann man also keine generelle Empfehlung ableiten. Weiterhin sind retrospektive Studien zum Dialysebeginn nur in sehr engen Grenzen aussagefähig, da alle eine signifikante methodische Einschränkung aufweisen: Patienten, die sich für die „späte“ Dialyse qualifizieren könnten, aber vor dem Dialysebeginn sterben oder sich vom ANV erholen, werden nicht erfasst ( $\bullet$ Abb. 1). Dies führt zu einem erheblich Selektionsbias. Es existieren in der Literatur auch Daten, dass die Einleitung eines Nierenersatzverfahrens die Letalität bestimmter Patienten eher verschlechterten kann - die Einleitung eines solchen Verfahrens ist auch mit Komplikationen behaftet (Antikoagulation, Gefäßzugang, etc.) $[17,45]$.

Antibiotikatherapie | Zusätzlich interferiert die Nierenersatztherapie nicht unerheblich mit der Dosierung einer Antibiotikatherapie. Es ist bekannt, dass die initiale adäquate Antibiotikathe-

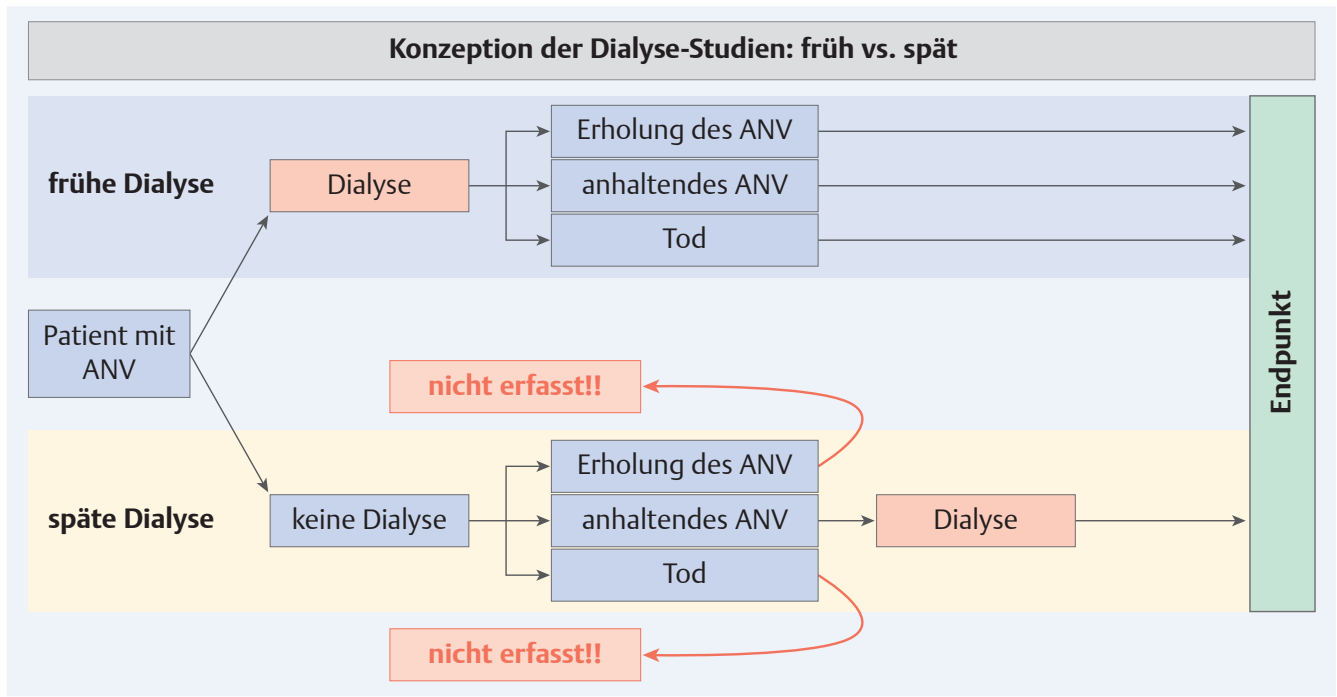

Abb. 1 Die Vergleichbarkeit von Studien, zum frühen vs. späten Dialysebeginn ist in mehrerer Hinsicht problematisch. Im Rahmen der späten Dialyse kann ein potenzieller Patient verstorben sein, bevor er dialysiert wird. Er wird dann in der Gesamtbetrachtung nicht erfasst. Aber auch Patienten, die ein reversibles ANV aufweisen, können sich spontan bessern, ohne dass diese dialysiert und erfasst werden. So entsteht insgesamt ein verzerrtes Bild und die verglichenen Patientengruppen unterliegen einem Bias. 


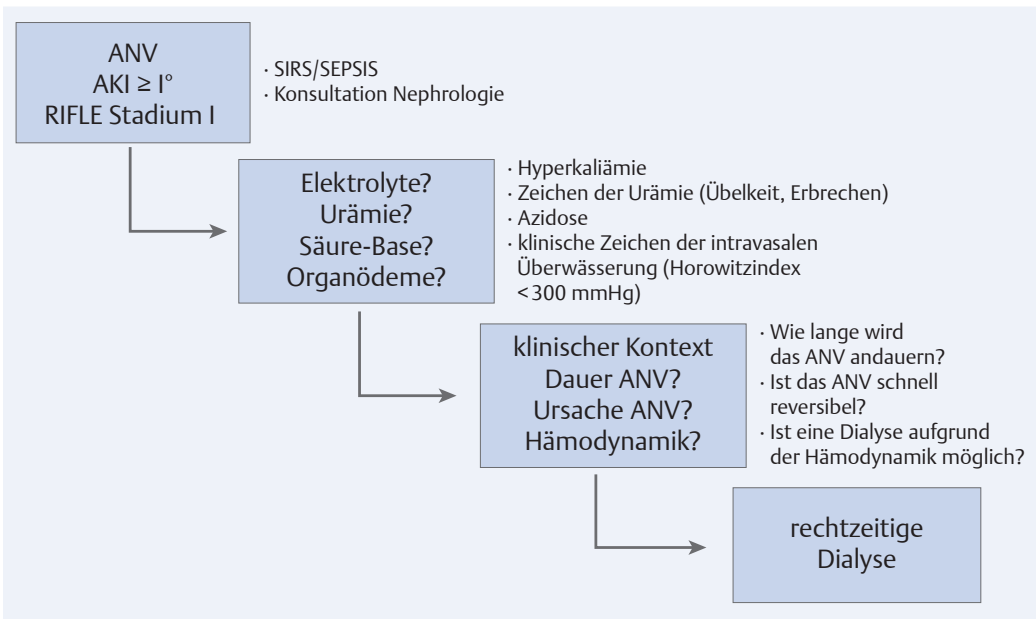

Abb. 2 Eine Dialyseindikation sollte frühzeitig als Teil der Sepsistherapie in Erwägung gezogen werden. Eine verzögerte Therapie, die zu Komplikation der Urämie führt, sollte unbedingt vermieden werden. Der Beginn einer Nierenersatztherapie soll sich dabei eng an den definierten therapeutischen Zielen orientieren.

rapie für die Prognose des Patienten entscheidend ist - so wird es in zahlreichen Leitlinien empfohlen [15]. Bei der Dosierung spielt nicht nur der Schweregrad des ANV eine Rolle, sondern auch die Art der Nierenersatztherapie. So konnte eine Reihe von Untersuchungen zeigen, dass die Antibiotika meistens zu niedrig dosiert sind:

Bei Dialyseverfahren mit den neueren Dialysatoren wird eine große Menge der Antibiotika entfernt.

Dies gefährdet die erfolgreiche Therapie des Patienten erheblich. Exemplarisch sei hier die Arbeit von Syler et al. erwähnt [41]: Die Autoren untersuchten an dialysepflichtigen septischen Patienten, ob die empfohlene Dosis der Breitspektrumantibiotika tatsächlich zu einer suffizienten Serumkonzentration führt, die die minimale Hemmkonzentration (MHK) für Pseudomonas erreichte. Zum Einsatz kamen

- Meropenem,

- Piperacillin/Tazobactam,

- Cefepim und

- Ceftazidim.

Die Ergebnisse der Untersuchung zeigten, dass Meropenem in $81 \%$, Piperacillin/Tazobactam in $71 \%$, Ceftazidim in nur 53\% der Fälle und Cefepim bei keinem Patienten die gewünschte MHK überschritt. Die Autoren kamen zu dem Schluss, dass die Antibiotika aufgrund der Nierenersatztherapie deutlich unterdosiert waren. Erschwerend kommt hinzu, dass genaue Angaben zur richtigen Dosierung bei der Dialyse für die einzelnen Dialysatoren fast vollständig fehlen. Dies macht eine adäquate antibiotische Therapie bei Patienten mit Nierenersatzverfahren nahezu unmöglich.

Frühe Dialyse von Vorteil I Basierend auf den Überlegungen zur Überwässerung profitieren Patienten mit einem ARDS und einem konsekutiv deutlich eingeschränkten Gasaustausch von einer frühen Dialyse [2]. Früh bedeutet hier ohne Manifestation der Überwässerung. Dies kann die

- Dauer der künstlichen Beatmung und auch

- die Wundheilungsstörung

positiv beeinflussen [47]. Zusätzlich leiden diese Patienten häufig an einer respiratorischen Azidose mit Hyperkapnie. Anstatt zu warten bis das Vollbild der Urämie und der renalen Azidose vorliegt, könnte also ein frühzeitiger Dialyseanschluss von Vorteil sein - auch unter der Vorstellung des gestörten Säure-Base-Haushalts durch das ANV. Eine retrospektive Erhebung unterstützt diese Theorie: So konnte von Ostermann et al. mit einer Analyse an 1847 Intensivpatienten herausarbeiten, dass der Zeitpunkt der Dialyse gemessen am pH bei einem ANV ein unabhängiger Risikofaktor für den Tod auf der Intensivstation ist [37]. Ein hoher pH war bei Dialysebeginn protektiv hinsichtlich der Mortalität. Eine therapierefraktäre Azidose vor Dialyse bei Ausbildung eines Multiorganversagens innerhalb von 48 h nach Dialysebeginn war ein Risikofaktore für den Tod auf der Intensivstation.

Präventive Dialyse I Aufgrund dieser Überlegungen sollte unserer Meinung nach die Indikation zur Dialyse nicht als reine „rescue Therapie“ zum Einsatz kommen - sondern vielmehr präventiv, um Komplikationen zu vermeiden. Tradierte Biomarker wie SCr und Harnstoff-N (BUN) sollten für die Indikationsstellung keine dominante Rolle mehr spielen. Wichtiger als ein Serumwert ist die gesamtklinische Beurteilung des Patienten, von der die Indikation für eine Dialyse abhängig gemacht werden sollte:

- Sepsis

- Multiple Organ Failure (MOF)

- Säure-Basenhaushalt

- Lungenfunktion

- Beatmungssituation, usw.

Volumenstatus als Kriterium | Eine klare Antwort, ob eine Dialyse „spät“ oder „früh“ eingeleitet werden sollte, kann man anhand der Daten nicht geben - derzeit ist nicht geklärt, wann überhaupt „spät“ oder „früh“ ist. Aber vielleicht ist dies auch die falsche Frage. Die Dialysebehandlung ist keine kurative Therapie. Sie ist lediglich eine supportive Therapie und sollte daher nur angewendet werden, wenn der Patient diese benötigt [27]. Was uns allerdings neben den Notfallindikationen für eine Dialyse fehlt, sind klare Kriterien, in welchen Fällen eine Dialyse benötigt wird und die Gesamtsituation für den Patienten verbessert. Aus den diskutierten Daten geht unserer Meinung nach hervor, dass dem Volumenstatus eine herausragende Bedeutung zukommt - ein Nierenersatzverfahren sollte unbedingt eingesetzt werden, um eine Überwässerung zu vermeiden ( $\mathbf{A b b}$. 2).

Interdisziplinäre Behandlung | Die Dialysebehandlung beim kritisch Kranken ist komplex. Deswegen sollten die Indikationsstellung und 
Durchführung einer Ersatztherapie interdisziplinär zwischen einem erfahrenen Nephrologen und Intensivmediziner erfolgen. Hierbei sollte folgendes diskutiert und interdisziplinär festgelegt werden:

- aktuelle Nierenfunktion

- Elektrolythaushalt

- Säure-Base-Haushalt

- Urämie

- Volumenstatus

- Prognose des ANV

- Medikamentendosierung (insbesondere Antibiotika)

- Kreislaufstabilität

- Art der Antikoagulation

Therapieziele formulieren | Darüber hinaus sollten lokale Standard Operating Procedures (SOPs) erarbeitet werden, um die Indikation zum Dialysebeginn festzulegen. Dabei sollte man die Gesamtsituation des Patienten und die lokalen Gegebenheiten berücksichtigen. Eine definitive Handlungsanweisung für den Beginn des Nierenersatzverfahrens ist aufgrund der Komplexität nicht möglich. Bei Unsicherheit hilft es aber, klare Ziele für die Therapie zu definieren, wie z.B.:

- Beseitigung einer Urämie

- negative Flüssigkeitsbilanz

- Reduktion des Kaliums, etc.

Sollten keine klaren Therapieziele vorliegen, ist ein abwartendes Vorgehen anzuraten.

Die Indikation zur Dialyse wird auch heute noch kontrovers diskutiert. Unserer Meinung nach sollte mit der Dialyse nicht gewartet werden bis die üblichen Notfallindikationen wie Diuretika resistentes Lungenödem oder das Vollbild der Urämie vorliegen. Wir schlagen vor, die Dialyse nicht als „Notfalltherapie“ anzusehen, sondern als Teil eines Gesamtkonzepts, um z. B. eine Überwässerung wirksam zu vermeiden. Man sollte das Nierenersatzverfahren aber auch nicht allzu leichtfertig einsetzen, da es mit Nebenwirkungen behaftet ist.

\section{Konsequenz für Klinik und Praxis}

- Die Mortalität eines dialysepflichtigen ANVs liegt bei bis zu $80 \%$ und ist somit eine besondere Herausforderung an die Intensivmedizin und Nephrologie.

- Die Überwässerung ist ein besonderes Problem: Die Dilution führt zu einem erniedrigten Kreatininwert, der die Diagnose verschleiert.

- Die Prognose des kritisch Kranken kann sich verschlechtern, wenn keine nephroprotektiven Maßnahmen eingeleitet und renal eliminierte Medikamente nicht an die tatsächliche Nierenfunktion angepasst werden.

- Darüber hinaus kann eine Überwässerung zu einer Perfusionsminderung der Niere mit unmittelbarer Schädigung führen.

\section{Literatur}

1 KDIGO. Clinical Practice Guideline for Acute Kidney Injury. Kidney Int Suppl 2012; 2: 1-138

2 The Acute Respiratory Distress Syndrome Network. Ventilation with lower tidal volumes as compared with traditional tidal volumes for acute lung injury and the acute respiratory distress syndrome. The Acute Respiratory Distress Syndrome Network. N Engl ] Med 2000; 342: 1301-1308

3 Basso F, Ricci Z, Cruz D et al. International survey on the management of acute kidney injury in critically ill patients: year 2007. Blood Purif 2010; 30: 214-220

4 Bellomo R, Cass A, Cole L et al. An observational study fluid balance and patient outcomes in the randomized evaluation of normal vs. augmented level of replacement therapy trial. Crit Care Med 2012; 40: 1753-1760

5 Bellomo R, Ronco C, Kellum JA et al. Acute renal failure - definition, outcome measures, animal models, fluid therapy and information technology needs: the Second International Consensus Conference of the Acute Dialysis Quality Initiative (ADQI) Group. Crit Care 2004; 8: R204-212

6 Bienholz A, Kribben A. KDIGO-Leitlinien zum akuten Nierenversagen. Nephrologe 2013; 8: 247-251

7 Bouchard J, Soroko SB, Chertow GM et al. Fluid accumulation, survival and recovery of kidney function in critically ill patients with acute kidney injury. Kidney Int 2009; 76: 422-427

8 Bouman CS, Oudemans-Van Straaten HM, Tijssen JG et al. Effects of early high-volume continuous venovenous hemofiltration on survival and recovery of renal function in intensive care patients with acute renal failure: a prospective, randomized trial. Crit Care Med 2002; 30: 2205-2211

9 Bradley SE, Bradley GP. The Effect of Increased Intra-Abdominal Pressure on Renal Function in Man. J Clin Invest1947; 26: 1010-1022

10 Carl DE, Grossman C, Behnke M et al. Effect of timing of dialysis on mortality in critically ill, septic patients with acute renal failure. Hemodial Int 2010; 14: 11-17

11 Cheatham ML. Abdominal compartment syndrome: pathophysiology and definitions. Scandinavian journal of trauma, resuscitation and emergency medicine 2009; 17: 10

12 Chertow GM, Burdick E, Honour M et al. Acute kidney injury, mortality, length of stay, and costs in hospitalized patients. J Am Soc Nephrol 2005; 16: 3365-3370

13 Chou YH, Huang TM, Wu VC et al. Impact of timing of renal replacement therapy initiation on outcome of septic acute kidney injury. Critical Care 2011; 15: R134

14 Cruz DN, Ricci Z, Bagshaw SM et al. Renal replacement therapy in adult critically ill patients: when to begin and when to stop. Contrib Nephrol 2010; 165: 263-273

15 Dellinger RP, Levy MM, Rhodes A et al. Surviving Sepsis Campaign: international guidelines for management of severe sepsis and septic shock, 2012. Intensive Care Med 2013; 39: 165-228

16 Doty JM, Saggi BH, Sugerman HJ et al. Effect of increased renal venous pressure on renal function. J Trauma 1999; 47: 1000-1003

17 Elseviers MM, Lins RL, Van der Niepen P et al. Renal replacement therapy is an independent risk factor for mortality in critically ill patients with acute kidney injury. Crit Care 2010; 14: R221

18 Feldkamp T. Akutes Nierenversagen-Pathophysiologie. Dialyse aktuell 2010; 14: 150-159

19 Feldkamp T, Bienholz A, Kribben A. Akutes Nierenversagen-Pathophysiologie und Klinik. Nephrologe 2011; 6: 113-119

20 Flori HR, Church G, Liu KD et al. Positive fluid balance is associated with higher mortality and prolonged mechanical ventilation in pediatric patients with acute lung injury. Crit Care Res Pract 2011; 2011: 854142

21 Goldberg R, Dennen P. Long-term outcomes of acute kidney injury. Adv Chronic Kidney Dis 2008; 15: 297-307

Vollständiges Literaturverzeichnis unter http://dx.doi.org/10.1055/s-0041-102253

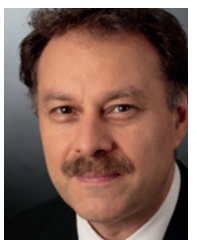

Prof. Dr. med. Fuat H. Saner ist Oberarzt an der Klinik für Allgemein-, Viszeral- und Transplantationschirurgie, Universitätsklinikum Essen fuat.saner@uni-due.de

Dr. med. Anja Bienholz ist wissenschaftliche Mitarbeiterin an der Klinik für Nephrologie, Universitätsklinikum Essen

anja.bienholz@uk-essen.de

Dr. med. Bartosz Tyczynski ist Oberarzt an der Klinik für Nephrologie, Universitätsklinikum Essen

bartosz.tyczynski@uk-essen.de

Prof. Dr. med.

Andreas Kribben

ist Direktor der Klinik für

Nephrologie, Universitäts-

klinikum Essen

andreas.kribben@uni-due.de

Prof. Dr. med.

Thorsten Feldkamp

ist Leitender Oberarzt an der

Klinik für Nieren- und

Hochdruckkrankheiten,

Universitätsklinikum Kiel

feldkamp@nephro.uni-kiel.de

Interessenkonflikt

T. Feldkamp gibt an, dass er Vortragshonorare und Stipendien von B. Braun und Fresenius erhalten hat. F. H. S., A. B., B. T. und A. K. geben an, dass kein Interessenkonflikt besteht.

DOI 10.1055/s-0041-102253

Dtsch Med Wochenschr 2015 . 140: 744-749

(c) Georg Thieme Verlag KG . Stuttgart · New York . ISSN 0012-0472 
22 Grams ME, Rabb H. The distant organ effects of acute kidney injury. Kidney Int 2012; 81: 942-948

23 Heung M, Wolfgram DF, Kommareddi M et al. Fluid overload at initiation of renal replacement therapy is associated with lack of renal recovery in patients with acute kidney injury. Nephrol Dial Transplant 2012; 27: 956-961

24 Hobson CE, Yavas S, Segal MS et al. Acute kidney injury is associated with increased long-term mortality after cardiothoracic surgery. Circulation 2009; 119: 2444-2453

25 Karvellas C], Farhat MR, Sajjad I et al. A comparison of early versus late initiation of renal replacement therapy in critically ill patients with acute kidney injury: a systematic review and meta-analysis. Crit Care 2011; 15: R72

26 Khwaja A. KDIGO Clinical Practice Guidelines for Acute Kidney Injury. Nephron Clin Pract 2012; 120: 179-184

27 Lameire N, Vanbiesen W, Vanholder R. When to start dialysis in patients with acute kidney injury? When semantics and logic become entangled with expectations and beliefs. Crit Care 2011; 15: 171

28 Lassnigg A, Schmidlin D, Mouhieddine M et al. Minimal changes of serum creatinine predict prognosis in patients after cardiothoracic surgery: a prospective cohort study. JASN 2004; 15: 1597-1605

29 Liu KD, Thompson BT, Ancukiewicz M et al. Acute kidney injury in patients with acute lung injury: impact of fluid accumulation on classification of acute kidney injury and associated outcomes. Critical Care Med 2011; 39: 2665-2671

30 Macedo E, Bouchard J, Soroko SH et al. Fluid accumulation, recognition and staging of acute kidney injury in critically-ill patients. Crit Care 2010; 14: R82

31 Mehta RL, Bouchard J, Soroko SB et al. Sepsis as a cause and consequence of acute kidney injury: Program to Improve Care in Acute Renal Disease. Intensive Care Med 2011; 37: 241-248

32 Mehta RL, Pascual MT, Soroko S et al. Spectrum of acute renal failure in the intensive care unit: the PICARD experience. Kidney Int 2004; 66: 1613-1621

33 Mikami O, Fujise K, Matsumoto S et al. High intra-abdominal pressure increases plasma catecholamine concentrations during pneumoperitoneum for laparoscopic procedures. Arc surg 1998; 133: 39-43

34 Mohmand H, Goldfarb S. Renal dysfunction associated with intra-abdominal hypertension and the abdominal compartment syndrome. JASN 2011; 22: 615-621

35 Murugan R, Karajala-Subramanyam V, Lee M et al. Acute kidney injury in non-severe pneumonia is associated with an increased immune response and lower survival. Kidney Int 2010; 77: 527-535
36 Newsome BB, Warnock DG, McClellan WM et al. Long-term risk of mortality and end-stage renal disease among the elderly after small increases in serum creatinine level during hospitalization for acute myocardial infarction. Arch Intern Med 2008; 168: 609-616

37 Ostermann M, Chang RW. Correlation between parameters at initiation of renal replacement therapy and outcome in patients with acute kidney injury. Crit care 2009; 13: R175

38 Payen D, de Pont AC, Sakr Y et al. A positive fluid balance is associated with a worse outcome in patients with acute renal failure. Crit Care 2008; 12: R74

39 Prowle JR, Echeverri JE, Ligabo EV et al. Fluid balance and acute kidney injury. Nat Rev Nephrol 2010; 6: 107-115

40 Saner FH, Cicinnati VR, Sotiropoulos G et al. Strategies to prevent or reduce acute and chronic kidney injury in liver transplantation. Liver Int 2012; 32: 179-188

41 Seyler L, Cotton F, Taccone FS et al. Recommended beta-lactam regimens are inadequate in septic patients treated with continuous renal replacement therapy. Crit Care 2011; 15: R137

42 Shiao CC, Wu VC, Li WY et al. Late initiation of renal replacement therapy is associated with worse outcomes in acute kidney injury after major abdominal surgery. Crit Care 2009; 13: R171

43 Shum HP, Chan KC, Kwan MC et al. Timing for initiation of continuous renal replacement therapy in patients with septic shock and acute kidney injury. Therapeutic apheresis and dialysis 2013; 17: 305-310

44 Uchino S. What is ,BEST' RRT practice? Contrib nephrol 2010; 165: 244-250

45 Vinsonneau C, Monchi M. Too early initiation of renal replacement therapy may be harmful. Crit Care 2011; 15: 112

46 Wald R, Quinn RR, Luo J et al. Chronic dialysis and death among survivors of acute kidney injury requiring dialysis. Jama 2009; 302: 1179-1185

47 Wiedemann HP, Wheeler AP, Bernard GR et al. Comparison of two fluid-management strategies in acute lung injury. N Engl J Med 2006; 354: 2564-2575

48 Wu VC, Ko WJ, Chang HW et al. Early renal replacement therapy in patients with postoperative acute liver failure associated with acute renal failure: effect on postoperative outcomes.JACS 2007; 205: 266-276 